\title{
Clinical Trial Patient Database Change Control
}

National Cancer Institute

\section{Source}

National Cancer Institute. Clinical Trial Patient Database Change Control. NCI Thesaurus.

Code C125438.

A document, report or log summarizing changes made to a clinical trial patient database. May include the reason for change, relevant approvals, impact/risk analysis, associated requirements, specifications and other documentation describing the validation and implementation of the change. 